\title{
Editorial :
}

\section{Dossier "PAISAJE Y RESILIENCIA URBANA"}

\author{
Por Leandro Varela
}

En la década de los 80 del siglo pasado, posteriormen te al retorno de la democracia en Argentina, la Universidad Nacional en su conjunto y la de La Plata en particular, retomó como base para reorganizarse institucionalmente las concepciones de la Reforma Universitaria. En ese contexto la Facultad de Arquitectura y Urbanismo se colocó a la vanguardia del proceso e impulsó el concurso de toda su planta docente y renovó el espíritu de la enseñanza disciplinar. En los años siguientes se profundizaron estas acciones ampliando su compromiso hacia los temas relacionados a la Investigación y la Extensión Universitaria.

En 1985 se constituyó el IDEHAB, Instituto de Estudios del Hábitat, ámbito donde se comenzaron a desarrollar y gestar las ramas de la investigación que se implementarían en las próximas tres décadas.

Dos investigadores de nuestra Facultad, Fernando Aliata y Graciela Silvestri, escribieron por entonces un libro fundante, "El Paisaje en las Artes y las Ciencias Humanas"1 que es uno de los primeros antecedentes en Argentina de los estudios multidisciplinares en estas temáticas, y que posteriormente sería ampliado conceptualmente en otro importante texto: "El Paisaje como cifra de Armonía"2 , sentando las bases para una ampliación del abordaje y debate disciplinar.

En 1995 el director del Instituto de Investigación Profesor Dr. Arq. Elias Rosenfeld y la Profesora Arq. Olga Ravella, conjuntamente con profesores de universidades de Europa y Chile crearon una red Internacional en la cual las problemáticas Urbanos ambientales se colocaron en el centro del debate.

Los miembros de la desde entonces denominada "Red Pehuén", luego de un par de reuniones en diferentes países, discutieron y diseñaron para su implementación en el año 1999 una formación de Posgrado con el grado de Magister llamada "Paisaje, Medioambiente y Ciudad". La misma se desarrolló durante dos años en la ciudad de Santiago de Chile y fue coordinada por la Arq. Franco- Chilena Adriana Araneda de la Escuela de Arquitectura de Paris la Villete en el marco del Programa Alfa de Cooperación Académica entre Europa y América Latina. De esa experiencia fundante formaron parte los docentes investigadores anteriormente mencionados a los cuales se sumaron un grupo de graduados con la intención de adiestrarse en las temáticas que la Red había considerado fundamentales en relación a las problemáticas relativas al paisaje, la ciudad y el territorio. Es así como la crisis ambiental en ciernes, el crecimiento desmesurado de las ciudades, la crisis energética ,los territorios transformados brutalmente por el extractivismo extremo, la globalización y la pérdida de

1. F.Aliata y G.Silvestri , El paisaje en el arte y las ciencias humanas. Colección Los fundamentos de las ciencias del hombre. Centro Editor de América Latina.1994 I.SB.N.:950-25-2179-x-

2. G.Silvestri y F.Aliata, El paisaje como cifra de armonía, Nueva Visión, Buenos Aires, 2001. Editorial: Nueva Visión I.S.B.N : 9506024308 
identidades regionales, entre otros temas, se plantearon como el objeto de estudio a ser abordado.

Por estos motivos, el grupo de la Red Pehuén en el marco del Programa Alfa Il decidió continuar esta experiencia formativa en la facultad de Arquitectura de la Universidad Nacional de La Plata poniendo en marcha la Maestría "Paisaje, Medioambiente y Ciudad" desde 2005 que continua funcionando hasta la actualidad. La misma tiene un gran número de egresados algunos de los cuales han seguido su formación en el Doctorado de Arquitectura y Urbanismo en esta institución.

Durante esos años se realizaron dos publicaciones que signaron esta nueva etapa. El primer libro fue "Paisaje, Reflexiones"3, del año 2002, compilación de los resúmenes de Tesis de las realizadas en la maestría desarrollado por los Becarios en la experiencia de Chile. El segundo libro, impreso posteriormente a la primera edición de la maestría en La Plata, "Diseñando el Paisaje"4 reunió los trabajos del Taller de Proyectos, con el objetivo de sintetizar el proceso proyectual que se planteó como estrategia de consolidación de un pensamiento complejo y pluridisciplinar que aun hoy seguimos perfeccionando.

El concepto de Paisaje cobra importancia en este debate a fines de las décadas de 1970/80 como mediador de la relación Cultura / Naturaleza. Un concepto que incorpora una nueva forma de ver las problemáticas mencionadas, mediadas a través de una óptica más amplia integradora e inclusiva. Un concepto amplio, proveniente del campo de la cultura y que durante los últimos años ha desarrollado y ampliado su corpus teórico para abordar las problemáticas integralmente.

A estas alturas podríamos decir también que dicho concepto ha cambiado profundamente. Ya no se concibe sólo como género pictórico, o el tradicional arte de hacer jardines, ni se operativiza en acciones solo a través del paisajismo. El proyecto de paisaje hoy en día se ha transformado en un conjunto de acciones e instrumentos que abordan la complejidad y consideran las problemáticas desde múltiples variables. Acciones e instrumentos que ya no provienen de un solo campo disciplinar, sino que reclaman y necesitan un trabajo conjunto de múltiples miradas y actores que confluyan en las respuestas a las problemáticas complejas.

Podemos encontrar en la web, numerosos proyectos e intervenciones que dan cuenta de este abordaje. Innumerables concursos internacionales, relativos a acciones urbanas y paisajísticas que dan cuenta de un acercamiento y concreción multivariable; y que a su vez se nutren conceptualmente de diversos campos disciplinares confluyentes.

Intervenciones que recuperan el cauce de los ríos urbanos siendo ganados para la ciudad como en diferentes ciudades de América Latina Europa y Asia, la intervención en humedales y áreas costeras degradadas, la recuperación de pasivos ambientales como canteras y tosqueras, la remediación y reconstrucción de las consecuencias de desastres naturales como Tsunamis, inundaciones y terremotos son algunos de los ejemplos que podemos mencionar en los últimos proyectos y obras desarrolladas a nivel mundial. Ejemplo de ello es el abordaje que desarrolla el estudio Chino, del Arq. KongjianYu denominado Turenscape, cuyos proyectos y obras están liderando el camino en diseño paisajístico ecológico. Fiel a la tradición de la cultura oriental el nombre Turenscape se deriva de dos palabras chinas: "Tu" (que significa "tierra") y "Ren" (que significa "gente"), combinado con la palabra en inglés "scape" para crear una palabra que indica la armonía entre tierra y gente. Es esta filosofía la que impulsa la creatividad detrás de la empresa multidisciplinaria y les permite crear paisajes que son innovadores a nivel ecológico y culturalmente significativo.

3. "Paisaje, Reflexiones" Laura Aón - Marcelo Bidinost - Victoria Goenaga - Devora Manuel - Oscar Michellod - Agustín Pinedo Gabriel Santinelli - Leandro Varela. Al Ediciones Al Margen, La Plata 2002

4. Diseñando el Paisaje, Colección Reflexiones sobre Paisaje. 
Podríamos decir que en términos vitruvianos el proyecto ya no tiene solo una mirada utilitaria, material y estética, sino que ahora pretende trascender el objetivo de armonía que se planteaba para la Arquitectura y busca constituirse en un agente de restauración de equilibrios perdidos.

A 20 años del comienzo de esa primera experiencia fundante en Chile y habiéndose consolidado el campo de estudios del paisaje en la FAU UNLP, el abordaje multidisciplinar del mismo con docentes e investigadores de diferentes unidades académicas, el desarrollo de la Maestría y el doctorado, es que proponemos la realización de esta publicación.

El Dossier "Paisaje y Resiliencia urbana" invita a reflexionar sobre cuál ha sido la evolución de la noción de paisaje en relación a problemáticas urbanas y periurbanas, sus modos de análisis (cualitativo y cuantitativo), de medición (indicadores, índices...), de representación (pintura, dibujo, cartografía, fotografía y video...) y como herramienta práctica proyectual (atlas, catálogo, plan de paisaje...) para el ordenamiento del territorio, así como cuál ha sido el grado de influencia del paisaje en la toma de decisiones de política pública, bajo qué instrumentos y normativas se enmarca y cómo desde el paisaje se podría contribuir a reducir la vulnerabilidad e incrementar la resiliencia urbana.

Invitamos a proponer avances o resultados de investigaciones científicas, desde una perspectiva integral, interdisciplinar, analítica esperando ampliar la discusión sobre un concepto complejo, a la vez tangible e intangible, que ha adquirido a través de los años nuevas formas de interpretación hasta ser reconocido en la actualidad como un elemento vital en la configuración urbana.

En base a estas premisas hemos recibido numerosos trabajos que han superado nuestras expectativas, incluso no todos han podido ser incorporados a este dossier. Aunque seguramente ya habrá forma de poder hacerlos públicos.

Los trabajos presentados abordan diferentes miradas y formas de considerar y tratar el paisaje, algunos desde un punto más analítico y científico, otros más enfocados a problemáticas que pueden ser encaradas desde el "proyecto de paisaje" y una tercer vertiente desde técnicas que permiten relevar y comunicar el paisaje ya sea desde operaciones tradicionales de representación o bien de técnicas avanzadas de análisis para llegar a propuestas.

El primer trabajo propuesto denominado “Del riesgo a la resiliencia. Hacia el diseño y gestión adaptativo del paisaje" da cuenta desde una perspectiva conceptual más ligada a las ciencias naturales, las problemáticas devenidas del "Cambio Climático" y las posibilidades de "riesgos" a las cuales se ven amenazadas hoy las ciudades y sus entornos. A partir de ello y partiendo de precisiones conceptuales como el de "resiliencia" plantea las posibilidades de abordaje de nuevas estrategias de planificación.

El segundo trabajo "Estudiar, Inventar y Conocer el Porto Marghera de Venecia" nos Ileva al conocimiento de los territorios industriales de la última mitad del siglo pasado y el estado actual de abandono como infraestructuras portuarias. En el caso particular abordado al diagnóstico se suma a la posibilidad de recuperación de áreas para una ciudad imposibilitada de crecer a la manera de otras. El exhaustivo método desarrollado e implementado para el conocimiento del lugar preludia posibilidades de abordaje proyectual desde un punto de vista urbano paisajístico.

Particularmente los temas relacionados al agua, y los sectores costeros son centrales en los artículos siguientes y es por ello que aparecen dos trabajos que abordan el mismo caso de estudio en la Ciudad de La Plata motivados por la grave inundación sucedida en el año 2013. El arroyo del Gato fue un epicentro de la tragedia socioambiental y su transformación se ha convertido en un tema central para ser abordado desde el proyecto de paisaje y la planificación urbana. 
En ese sentido el trabajo denominado "El paisaje detrás de las diagonales. Vínculos entre arroyos y urbanización en la ciudad de La Plata" realiza un relevamiento histórico sobre la correspondencia entre los aspectos naturales donde se emplazó el trazado fundacional de la ciudad y sus consecuencias actuales en relación a las inundaciones. Un trabajo de enfoque cualitativo, que profundiza desde una perspectiva histórica, las vinculaciones que fueron estableciéndose desde los primeros registros entre paisaje fluvial y urbanización en la región, haciendo foco en la cuenca del arroyo El Gato y su conexión con el borde urbano del casco fundacional de la ciudad.

Por otra parte "Paisajes Resilientes" Lineamientos para un proyecto de paisaje en el arroyo El Gato en pos de incrementar la resiliencia urbana, complementariamente al trabajo anterior se posiciona en un enfoque más direccionado al, "¿qué hacer?" desde el punto de vista proyectual. Determinar el grado de vulnerabilidad de la cuenca de El Gato es su objetivo, con el fin de incrementar la resiliencia urbana mediante el proyecto "Paisajes resilientes". El mismo propone reinterpretar la relación agua-ciudad a través de un proyecto de paisaje integrador y participativo en la cuenca media del arroyo intentando generar pautas de intervención generalizables en la región.

Por último se presentan dos trabajos que si bien no presentan al Paisaje y la Resiliencia Urbana como centro de su planteo, hemos decidido incorporarlos como parte de este dossier por entender que las representaciones en todas sus formas son parte inescindibles de la problemática y que su implementación es parte de los métodos para enfrentar el problema.

Así, el trabajo "La dimensión temporal en la representación gráfica de espacios urbanos y proyectos de paisaje" nos propone reflexionar sobre el tiempo y las formas de graficar los cambios estacionales de los componentes principales del paisaje. Como dice su autor "La representación de un proyecto de paisaje debe tener en cuenta esta condición de muta $\neg$ bilidad para comunicarla de manera adecua $d$ da. No obstante, debemos considerar que los diferentes sistemas codificados de proyección solo pueden representar las tres dimensiones del espacio cartesiano. Esa limitación inherente obliga a estudiar métodos alternativos que per-mitan introducir la variable tiempo, entendida como la cuarta dimensión a ser representada. A partir de esta premisa podemos considerar que no solo captar el tiempo y plasmarlo en una gráfica determinada es una premisa en si misma sino que puede incluirse en los proyectos de paisaje para dar cuenta de los procesos de recuperación de espacios urbanos y sus evoluciones.

Por último incorporamos el trabajo "Paisaje urbano, infraestructura ecológica y regulación de la temperatura. El caso de la ciudad de Mar del Plata, Argentina" dando cuenta de los estudios científicos que pueden ser la apoyatura de análisis y el aporte de miradas para conclusiones y líneas de acción del proyecto urbano y de paisaje. La infraestructura ecológica urbana conceptualmente aporta la mirada interdisciplinaria que se requiere para entender los procesos de la ecología urbana y sus consecuencias en las dinámicas de organización y configuración de la ciudad. El uso de herramientas gráficas y tecnológicas sumadas a metodologías de análisis, puede brindar la información necesaria para generar espacios urbanos más confortables y adecuados para su habitabilidad y disfrute, y por otra parte anticipar algunas situaciones que podrían ocasionarse relacionadas al cambio climático.

De esta forma y a través de esta selección de artículos queremos dar cuenta de una problemática cada día más relevante en los estudios urbanos y de paisaje. Las temáticas desarrolladas son representativas de dificultades comunes a las cada vez más complejas pobladas y extensas ciudades estudiadas.

Por otro lado creemos que expresan muy claramente la necesidad del trabajo interdisciplinar en el abordaje de los problemas, en la búsqueda de soluciones integrales y no sectoriales a las cuales 
estamos acostumbrados y que sabemos no han dado los resultados que se esperaban para la resolución de las mismas.

Creemos que no se completa con estos textos la visión integral necesaria y multidisciplinar para poder abarcar el abanico de problemáticas a las cuales el concepto y proyecto de paisaje, conjuntamente con el proyecto urbano deberían atender. Pero sabemos que estamos haciendo un pequeño aporte a la generación de conciencia de esto que planteamos y es por ello que esperemos haber cumplido mínimamente con el propósito que nos habíamos propuesto y dejar trazada la posibilidad de seguir trabajando e intercambiando conocimientos con profesionales interesados en elaborar conjuntamente estos abordajes cada día más urgentes, necesarios e imprescindibles. 